\title{
Elevated plasma concentration of vascular endothelial growth factor in cardiac myxoma
}

\author{
Kenneth R. Bennett, MD, Jian-Wei Gu, MD, ${ }^{\text {b }}$ Thomas H. Adair, PhD, ${ }^{b}$ and Bobby J. Heath, MD, ${ }^{\dagger}{ }^{\dagger}$ Jackson, Miss
}

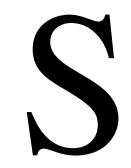

hortly after Kono and associates ${ }^{1}$ reported their findings concerning the role of vascular endothelial growth factor (VEGF) gene expression in the development of cardiac myxomas, we had the opportunity to assay plasma VEGF before and after surgery in a 31-year-old black woman with multicentric recurring myxomas. At the age of 10 years, she had undergone excision of a left atrial myxoma. In the following decade, she had 4 more operations with excision of 19 myxomas. The tumors ( 9 on the right side and 10 on the left side), occurring at widely separated and random sites within both atria and on the tricuspid and mitral valves, appeared to develop de novo without invasion of surrounding tissue. The sizes ranged from a "nodule" to $3 \times 4 \mathrm{~cm}$, and the histologic features, by both light and electron microscopy, were typical for myxoma. Now, 12 years after her fifth heart operation, the tumor recurred and she underwent a sixth operation. We report our observations.

\section{Methods}

Three tumors were resected. The largest $(7.0 \times 4.9 \times 1.2 \mathrm{~cm})$ arose from the floor of the right atrium between the inferior vena cava

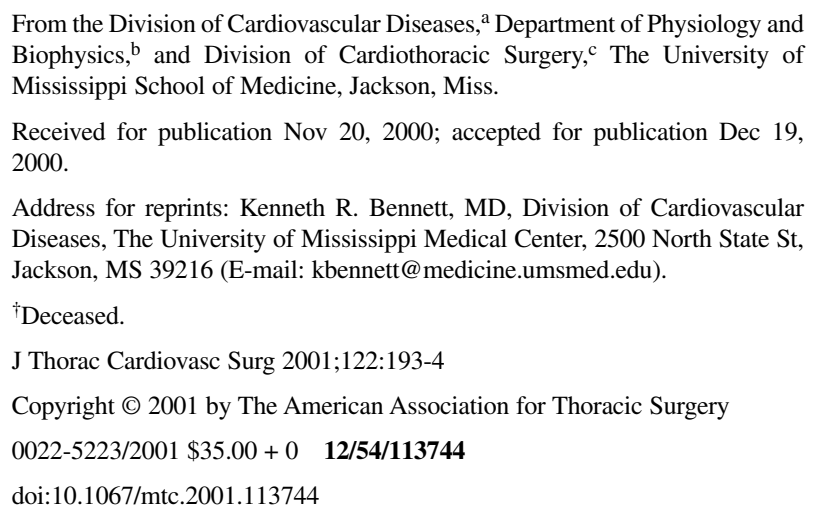

Address for reprints: Kenneth R. Bennett, MD, Division of Cardiovascular Diseases, The University of Mississippi Medical Center, 2500 North State St, Jackson, MS 39216 (E-mail: kbennett@medicine.umsmed.edu).

${ }^{\dagger}$ Deceased.

J Thorac Cardiovasc Surg 2001;122:193-4

Copyright $\odot 2001$ by The American Association for Thoracic Surgery

$0022-5223 / 2001 \$ 35.00+0 \quad \mathbf{1 2 / 5 4 / 1 1 3 7 4 4}$

doi:10.1067/mtc.2001.113744

and the os of the coronary sinus; a second tumor $(4.0 \times 2.0 \times 2.5$ $\mathrm{cm})$ was attached to the ventricular septum 1 to $2 \mathrm{~cm}$ below the pulmonary valve; and the third and smallest $(0.7 \times 0.6 \times 0.2 \mathrm{~cm})$ arose from the dome of the left atrium. Blood for VEGF assay was collected 2 to 3 hours before the operation and then 2 days, $5 \frac{1}{2}$ weeks, and 6 months after the operation.

Plasma concentration of VEGF was compared with that of 4 men aged $41 \pm 9$ years, weighing $77 \pm 16 \mathrm{~kg}$, who had normal blood pressure and who presumably were free of cardiovascular disease, peripheral vascular disease, and malignancy.

The samples were collected with ethylenediaminetetraacetic acid, immediately centrifuged, and the plasma was stored at $-70^{\circ} \mathrm{C}$. The plasma concentration was determined by means of sandwich enzyme-linked immunosorbent assay (R\&D Systems, Minneapolis, Minn). ${ }^{2}$

This study was conducted in accordance with the guidelines of the Institutional Review Board of The University of Mississippi Medical Center. The subject of this study gave informed consent.

\section{Results}

There was a marked fall in the VEGF level after the operation. From a preoperative level of $83.1 \mathrm{pg} / \mathrm{mL}$, it fell to $23.4 \mathrm{pg} / \mathrm{mL}$ at 2 days, $20.7 \mathrm{pg} / \mathrm{mL}$ at $5 \frac{1}{2}$ weeks, and $32.0 \mathrm{pg} / \mathrm{mL}$ at 6 months. The plasma concentration of VEGF in 4 healthy subjects (mean \pm SD) was $40.0 \pm 26.4 \mathrm{pg} / \mathrm{mL}$.

\section{Discussion}

VEGF, also known as vascular permeability factor, is a glycoprotein that initiates mitosis of endothelial cells. Although an expected plasma constituent, there is no established "normal" concentration, and from our observations the range is wide. The patient's plasma level before removal of the tumors was $25 \%$ above the highest found in the 4 healthy men; although "high," it is not conclusive. However, the marked fall (from 83.1 to $23.4 \mathrm{pg} / \mathrm{mL}$ ) noted 2 days after the operation and persisting at $5 \frac{1}{2}$ weeks and at 6 months suggests the level was heightened as a consequence of active expression of VEGF limited to the tumor; that supposition is 
in accord with the findings of Kono and associates. ${ }^{1}$ They analyzed myxomas and adjacent atrial myocardium from 15 patients, each with a solitary tumor (13 left atrium, 2 right atrium) for VEGF protein expression and microvessel density. All tumors showed a positive immunohistochemical reaction for VEGF, whereas the adjacent atrial myocardium exhibited a negative reaction, indicating that expression of the VEGF gene was confined to the tumor. Tumors with the highest expression of VEGF showed the greatest density of microvessels (angiogenesis), suggesting that it is VEGF expression by the myxoma that induces and sustains angiogenesis for tumor growth.

Although expression of VEGF is characteristic of myxomas, there is the question of sensitivity. What amount of tumor tissue and/or gene expression is necessary to lift the plasma concentration of VEGF to a meaningful level? Not only was this woman's tumor multicentric, involving both sides of the heart, but the aggregate tumor mass was greater than usually present with the typical solitary myxoma. Otherwise, little more can be made of this single case. However, should it be established that a heightened concentration of plasma VEGF is characteristic of myxomas, such knowledge might well lead to advances in diagnosis, management, follow-up, and perhaps novel therapies to inhibit angiogenesis. ${ }^{3}$

\section{References}

1. Kono T, Koide N, Hama Y, Kitahara H, Nakano H, Suzuki J, et al. Expression of vascular endothelial growth factor and angiogenesis in cardiac myxoma: a study of fifteen patients. $J$ Thorac Cardiovasc Surg. 2000;119:101-7.

2. Gu JW, Adair TH. Hypoxia-induced expression of VEGF is reversible in myocardial vascular smooth muscle cells. Am $J$ Physiol. 1997;273:H628-33.

3. O'Reilly MS, Boehm T, Shing Y, Fukai N, Vasios G, Lane WS, et al. Endostatin: an endogenous inhibitor of angiogenesis and tumor growth. Cell. 1997;88:277-85. 